\title{
BMI open Protocol for the Northern Manhattan Diabetes Community Outreach Project. A randomised trial of a community health worker intervention to improve diabetes care in Hispanic adults
}

To cite: Palmas W, Teresi JA, Findley S, et al. Protocol for the Northern Manhattan Diabetes Community Outreach Project. A randomised trial of a community health worker intervention to improve diabetes care in Hispanic adults. BMJ Open 2012;2 e001051. doi:10.1136/ bmjopen-2012-001051

- Prepublication history and additional materials for this paper are available online. To view these files please visit the journal online (http://dx. doi.org/10.1136/ bmjopen-2012-001051).

Received 20 February 2012 Accepted 27 February 2012

This final article is available for use under the terms of the Creative Commons Attribution Non-Commercial 2.0 Licence; see

http://bmjopen.bmj.com

${ }^{1}$ Department of Medicine, Columbia University, New York, New York, USA

${ }^{2}$ Hebrew Home for the Aged, Riverdale, New York, USA

${ }^{3}$ Joseph Mailman School of Public Health, Columbia University, New York, New York, USA

${ }^{4}$ Alianza Dominicana, New York, New York, USA ${ }^{5}$ Department of Medicine, University of Miami, Miami, Florida, USA

Correspondence to Dr Walter Palmas; wp56@columbia.edu

\section{ABSTRACT}

Objective: Hispanics in the USA are affected by the diabetes epidemic disproportionately, and they consistently have lower access to care, poorer control of the disease and higher risk of complications. This study evaluates whether a community health worker (CHW) intervention may improve clinically relevant markers of diabetes care in adult underserved Hispanics.

Methods and analysis: The Northern Manhattan Diabetes Community Outreach Project (NOCHOP) is a two-armed randomised controlled trial to be performed as a community-based participatory research study performed in a Primary Care Setting in Northern Manhattan (New York City). 360 Hispanic adults with poorly controlled type 2 diabetes mellitus (haemoglobin A1c $>8 \%$ ), aged 35-70 years, will be randomised at a 1:1 ratio, within Primary Care Provider clusters. The two study arms are (1) a 12-month CHW intervention and (2) enhanced usual care (educational materials mailed at 4-month intervals, preceded by phone calls). The end points, assessed after 12 months, are primary = haemoglobin A1c and secondary $=$ blood pressure and low-density lipoprotein-cholesterol levels. In addition, the study will describe the $\mathrm{CHW}$ intervention in terms of components and intensity and will assess its effects on (1) medication adherence, (2) medication intensification, (3) diet and (4) physical activity. Ethics and dissemination: All participants will provide informed consent; the study protocol has been approved by the Institutional Review Board of Columbia University Medical Center. CHW interventions hold great promise in improving the wellbeing of minority populations who suffer from diabetes mellitus. The NOCHOP study will provide valuable information about the efficacy of those interventions vis-à-vis clinically relevant end points and will inform policy makers through a detailed characterisation of the programme and its effects.

Clinical trial registration number: NCT00787475 at clinicaltrials.gov.

\section{ARTICLE SUMMARY}

Article focus

- Randomised controlled trial.

- CHW intervention.

- Diabetes care.

Key messages

- This community-based participatory research study is a collaboration between a community organisation and a university in Northern Manhattan, New York City.

n The goal is to assess whether the CHW worker intervention may improve diabetes care in underserved adult Hispanics from the community.

- The primary outcome of interest is haemoglobin A1c, a marker of diabetes control; secondary outcomes are blood pressure and low-density lipoprotein cholesterol levels.

Strengths and limitations of this study

- This study will examine effects of the CHW intervention after 12 months, a longer time period than in previous studies.

- The CHW intervention protocol was developed in a culturally appropriate manner to address the needs of Hispanics residing in our community.

- If proven efficacious, it will warrant examination in other cultural socioeconomic milieus.

\section{INTRODUCTION}

The current diabetes epidemic affects US Hispanics disproportionately, both in prevalence and frequency of complications as compared with Caucasians. ${ }^{1}$ Hispanics suffer from less access to care and poorer control of their diabetes. ${ }^{23}$ On the other hand, there is a great paucity of culturally appropriate models of care that maximise access and enhance healthcare delivery in Hispanics. ${ }^{4}$ The lack of such models perpetuates the role of language and culture as 'barriers to care'. 
Thus, there is great interest in patient-cantered interventions that, instead, embrace the culture and language of our patients. This paradigm shift has the potential to transform those perceived 'barriers' into integral components of the intervention, empowering patients and their families in dealing with diabetes mellitus and helping them navigate the complexities of our medical system.

Community health workers (CHWs, known as Promotoras or Promotores de Salud in Spanish) have been shown to be efficacious in improving healthcare delivery around the world, including Latin America and the USA. ${ }^{6}$ However, a better characterisation of the efficacy of CHW interventions is needed, particularly in regards to widely accepted clinical end points, such as serum haemoglobin A1c (A1c, a measure of blood glucose levels over the previous weeks), blood pressure and cholesterol levels. Several randomised controlled trials (RCTs) have been performed to assess the potential benefits of CHW interventions in improving the care of minority populations with diabetes. However, those trials recruited relatively small numbers of participants and were carried out for rather short periods of time. ${ }^{7-13}$ Indeed, as recently reviewed, data from some of those studies suggest that short-term efficacy achieved in lowering A1c is later lost at 12 months. ${ }^{14}$ Systematic reviews and meta-analyses have also highlighted the need for larger and longer term RCTs in Hispanic and other underserved communities. ${ }^{14-17}$ In addition to clinically relevant end points (such as A1c lowering), there is a clear need for interventions that are well described both in nature and intensity and that target a well-defined population. ${ }^{17}$

We describe here the protocol of a study designed to address those gaps in knowledge, the Northern Manhattan Diabetes Community Outreach Project (NOCHOP). The NOCHOP is a RCT to evaluate the efficacy of a 12-month CHW intervention to improve the care of Hispanics with poorly controlled type 2 diabetes mellitus. It is a two-arm study with an active control group, designed such that the control group receives enhanced standard care. It is a community-based participatory research enterprise designed and conducted by two partner institutions from Northern Manhattan: Alianza Dominicana, Inc., and Columbia University.

\section{METHODS AND ANALYSIS \\ Study outcomes}

The primary study outcome is glycaemic control, measured by A1c. The secondary outcomes are systolic and diastolic blood pressure and low-density lipoprotein (LDL) cholesterol levels. We also are collecting data, which will allow examination of the putative mechanisms that may account for the hypothesised effects of the intervention in this population. Data are being collected during the intake and 1-year follow-up examination on the following mechanistic end points: (1) medication adherence, (2) medication intensification, (3) diet and (4) physical activity.

\section{Study participants}

NOCHOP is an RCT of 360 Hispanic participants with poorly controlled type 2 diabetes, aged $35-70$ years and who are currently receiving care at one of our primary care practice sites in Northern Manhattan. ${ }^{18}$ Participants are classified as having poorly controlled diabetes if their last A1c measurement (performed in the preceding 12 months) was $\geq 8.0$. Exclusion criteria are (1) type 1 diabetes and/or diabetes with onset before age of 25; (2) subjects who do not self-identify as Hispanic; (3) any lifethreatening or extreme medical comorbidity, such as an active cancer or end-stage cardiopulmonary disease; (4) having a diabetes diagnosis for $<1$ year; (5) planning to move out of the neighbourhood during the next year; (6) enrolment in any other study and (7) arm circumference of $>47 \mathrm{~cm}$ (due to inability to accurately measure blood pressure using an oscillometric device).

All participants provide informed consent prior to enrolment; the study protocol has been approved by the Institutional Review Board (IRB) of Columbia University Medical Center. Consent is obtained by trained study personnel, following IRB-approved procedures. Recruitment is performed within the Primary Care Clinics at Columbia University Medical Center, and it is centred around Primary Care Physicians (PCP), who approve all contacts with potential participants. This approach has proven successful for our group when recruiting, from the same population, for a recent RCT of innovative diabetes care management. ${ }^{19}$ Moreover, the collaboration with Alianza Dominicana, the largest and bestknown community organisation in our neighbourhoods, probably enhances recruitment.

Participant attrition is usually a concern in trials enrolling underserved populations, as it may compromise the statistical power of the study. Thus, steps are taken in both arms to maximise retention. In the intervention arm, CHWs regularly stress the importance to all participants of undergoing their 12-month evaluation visit, and they use reminders when approaching the end of the intervention. In the control arm, the study coordinator takes the opportunity provided by the scheduled phone calls (see below) to address this issue. Based on the intention-to-treat principle, all efforts will be made to bring participants back for the 12-month evaluation visit. For those participants who are unable or unwilling to undergo that evaluation, sensitivity analyses will be performed-please see the Statistical analysis methods section, for a description of our analytic approach to missing data. Of note, as described in the supplementary data file, our sample size was determined using a conservative approach to attrition when modelling the statistical power estimates.

\section{Randomisation}

After providing informed consent, participants are remotely and blindly randomised (1:1) to either 
intervention (CHW intervention for 12 months) or to enhanced usual care (EUC) by the Research Department at the Hebrew Home at Riverdale, NY. Randomisation is clustered within PCP practice; the algorithm accounts for rolling enrolment within PCPs. Balance between the two study arms is checked periodically.

\section{Procedures}

All participants undergo two examination visits: one at baseline and another one at the end of the participation a year later. Personnel performing evaluation visits are blinded to randomisation status. Subjects are instructed to come to examinations fasting, and having held their diabetes medications, but taking their blood pressure medications. Questionnaire data are obtained during examination visits using a computer-assisted telephone interviewing system. ${ }^{20}$

\section{Measures}

Height and weight are measured without shoes and wearing lightweight clothes and using a stadiometer and a validated digital scale. All measurements are recorded to the nearest $0.1 \mathrm{~cm} / 0.1 \mathrm{~kg}$. Specimens are analysed in blinded fashion by the Columbia University CRC Core Laboratory. A1c is measured using a latex agglutination assay (Hitachi 912 Polymedco Inc., Cortlandt Manor, New York, USA). Total cholesterol, triglyceride and highdensity lipoprotein cholesterol is measured using enzymatic colorimetric methods (Vitros, Johnson \& Johnson, New Brunswick, New Jersey, USA). LDL cholesterol is calculated using the Friedewald equation. ${ }^{21}$ For subjects with triglyceride level $\geq 300 \mathrm{mg} / \mathrm{dl}$, LDL cholesterol is measured directly using a homogeneous assay (Polymedco, Cortlandt Manor, New York, USA). Resting blood pressure is measured using a BpTRU automated oscillometric device (VSM Tech Ltd, Coquitlam, BC, Canada). Three measurements are obtained following 5 min of rest. The average of the second and third measurements is recorded as the resting blood pressure. Other constructs measured include medication adherence, ${ }^{22}$ dosage and intensity, ${ }^{23}$ physical activity, ${ }^{24}$ diet $^{25}$ and depression. ${ }^{26}$

\section{CHW intervention}

\section{Basic features}

The CHW intervention is based on (1) existing consensus of successful diabetes interventions in vulnerable populations and (2) its promise as a sustainable generalisable intervention. Two full time CHWs based at Alianza Dominicana, Inc., are delivering a multicomponent intervention that includes home visits, group visits and telephone follow-up, the focus of the home visits is on assessment of existing barriers to healthcare (diabetes and non-diabetes), empowering the patient to overcome these barriers and then developing achievable goals for the upcoming year. The group visits focuses on nutrition and exercise activities. The phone intervention serves as a follow-up mechanism for adherence to the individualised plan and reinforcement. The intervention is summarised in figure 1 of the supplementary data. CHW intervention is flexible and tailored to each participant's needs, but the goal will be to perform at least four home visits, 10 group sessions and 10 follow-up phone calls per subject over a 12-month period.

\section{CHW medical service/patient navigator activities}

Self-management is key to the CCM, and fundamental to this is empowering patients to make effective use of the healthcare system. ${ }^{27} 28$ This requires a positive and productive relation with the PCP, wherein the patient is comfortable in asking questions and speaking honestly about concerns regarding medications. ${ }^{29}{ }^{30}$ Through individual and group activities, the CHWs help the participants develop communication and self-advocacy skills to be able to take a more active role in their visits. They teach participants how to maximise their time with the provider, how to advocate for themselves and what questions they should ask. Additional patient navigator activities by CHWs during the home visit may include reminding patients of their next appointments and, if needed, setting up home medication reminder systems for patients such as refrigerator charts noting when it is time to get refills.

\section{Referrals}

The CHWS also assist the participants in accomplishing their goals by connecting them to needed services. The CHWS make referrals to community-based resources, both for social and healthcare services. One example would be if the participant is facing eviction or experiencing problems such as domestic violence. Alianza has specific programmes for housing and domestic violence and the CHWS facilitate referrals; if not available through Alianza, they are referred to other community partners where such services would be available.

\section{Informatics support for CHWs}

The CHWs have remote real-time access to participant data, in a HIPAA-compliant manner, ${ }^{31}$ through secure access to a dedicated database.

\section{EUC arm of the study}

Patients randomised to the control group receive usual care from their PCP. This includes routine monitoring and care from the PCP and the possibility of referral to several existing diabetes management resources, among them the multidisciplinary comprehensive Naomi Berrie Diabetes Center at Columbia University Medical Center. The N. Berrie Center resources comprise a multidisciplinary team of endocrinologists, certified diabetes educators and nurse case managers who provide intensive diabetes case management requiring very involved patient participation. Providers can also refer patients to the Visiting Nurse Service of New York. The Visiting Nurse Service programme includes nurses, nutritionists and diabetes educators who can deliver home-based diabetes care and education to patients. This service is ideal for those older patients who are home-bound. In addition, our primary care clinic has a certified diabetes 
health educator nurse who provides on site individual diabetes education, group diabetes classes and, if requested, can assist providers with case management. In NOCHOP, PCPs remain free to use any of these existing resources for their patients, at their discretion. The usual care received will be enhanced by providing patients with three sets of Spanish language educational materials published by the National Institutes of Health. These materials include information on communication between physician and patient, diabetes management, mental health and a diabetes cookbook. During the 12month period, a project coordinator calls EUC patients four times. The goals of the phone calls are to (1) ensure that the participants have received the mailed brochures and that they find those brochures appropriate for their own literacy and (2) maximise retention in the study, aiming to reduce attrition in this group.

\section{STATISTICAL ANALYSIS METHODS}

\section{Sample size and power}

The sample size was chosen to ensure sufficient power to detect clinically meaningful effects associated with a change in A1c as a continuous outcome. The calculations performed indicate that the proposed sample size of 360 ( 180 per arm) will provide sufficient power for the main study hypotheses, under a variety of assumptions. The power calculations, including a detailed description of assumptions and scenarios, are described in detail in the supplementary file.

\section{Unit of analysis and clustering}

The patient will constitute the unit of analysis for the study. Patients will be randomised within PCP; therefore, sample sizes must be larger to account for unreliability of measures and for design features. ${ }^{32-34}$ Both correlation among repeated measures over time on the same subject and correlation due to clustering of patients within providers (characteristics of the providers or practice which may influence outcomes among their patients) will be taken into account. This dependency among members of the cluster will inflate the variance of the effect of the intervention. ${ }^{35}$

\section{Adjustment for multiple comparisons}

Adjustment for multiple comparisons is an area of controversy. ${ }^{36-40}$ Following recent guidelines for clinical trials, ${ }^{41}$ we propose to treat primary and secondary outcomes as separate clusters, setting a 0.05 level of significance to the primary outcome within each cluster. A Bonferroni or Benjamini-Hochberg correction would be applied to secondary treatment outcomes. Thus, a 0.05 level will be assigned to the A1c outcome and a 0.01 level to the three secondary outcomes (LDL, diastolic blood pressure and systolic blood pressure).

\section{Analyses}

A parallel group design with equivalent baseline values as a result of randomisation is proposed. Calculations are provided for the primary outcome treated as continuous and as binary. The main analyses will be performed using continuous data. Analyses of A1c treated as binary assumes intent-to-treat and that all dropout and missing data are considered as failures. Thus, the proportion of successes is based on the entire sample randomised. However, in the context of cost limitations, large samples are required to examine small effects when continuous data are treated as binary because of the severe loss of power associated with dichotomising a normally distributed dependent variable such as Alc. Dichotomisation of skewed variables leads to even greater loss of power. ${ }^{42}$ Additionally, there are theoretical considerations that include the fact that in health disparities research, stringent goals, for example, complete glycaemic control, may not be realised and one may consider smaller average reductions in outcomes as clinically meaningful.

The general approach to the analyses is guided by our own experience in the analyses of such data ${ }^{43} 44$ and by recent reviews of best methods for analyses of longitudinal data from clinical trials. ${ }^{45}$ Because the design is to randomise individuals to groups within PCP strata, some baseline imbalance in the outcome might occur; in this case, the basic analytic approach will be analysis of covariance (ANCOVA) model that adjusts for baseline values of the outcome, as well as for the design feature of clustering. In order to determine the best approach, two basic models could be examined. One is a basic t test or ANCOVA approach, with inclusion of a random effect for PCP to model the clustered data. The second is a repeated measures approach that examines time as continuous. The latter allows inclusion of more subjects, however, with only two waves of data (and if $90 \%$ of the subjects are interviewed within \pm 2 months of the 12 month mark), it is not clear that there will be sufficient benefits associated with the approach. The post-treatment values of continuous outcomes will be modelled as functions of baseline values, treatment and the interaction of baseline and treatment. A general longitudinal mixed effects model, using SAS PROC MIXED, will be used to allow for the correlation between subjects within a PCP. Additionally, the group heterogeneity in cluster and residual variances may require modelling to satisfy model assumptions and improve model fit. (There may be violations of the more rigid assumptions involved in ANCOVA, such as homoscedasticity, so that modelling the group heterogeneity in cluster and residual variances will be necessary.) Based on prior analytic experience with the outcome variables, the need to transform them is not anticipated. Although the primary analysis is to examine A1c as a continuous measure, it is also proposed to treat A1c as a binary outcome defining those with poor glycaemic control as A1c $\geq 9.0$. In this case, dichotomous outcome measures will be analysed using generalised estimating equations to account for potentially correlated outcomes of subjects with the same PCP (PROC GLIMMIX in SAS). Prior to analyses, 
baseline values of all variables from each arm will be examined; however, no $\mathrm{p}$ values will be provided, and covariates (other than baseline values) are not proposed for inclusion in the main analyses of treatment effects. Examination of baseline differences on key variables between subjects remaining and those lost to follow-up will also be conducted. The first set of analyses will not adjust for dropout. Only cases with complete data will be included; however, as stated, these analyses will include those who did not complete the CHW intervention but who returned to provide the follow-up interview, under an intent-to-treat design. The intent-to-treat analyses of the total group could be repeated using baseline values carried forward to account for cases lost to follow-up (using SAS PROC MIXED). However, baseline values carried forward is not optimal, depending on the type of variable studied. ${ }^{46}$ For example, blood pressure may increase over time due to ageing. Thus, other methods of examining missing data, for example, propensity scores, EM algorithm and multiple imputation sensitivity analyses, will be considered.

\section{ETHICS AND DISSEMINATION}

Data and Safety Monitoring Board

The Data and safety monitoring board (DSMB, also known as a Data Monitoring Committee) is an independent four-member multidisciplinary group consisting of biostatisticians and clinicians that collectively have experience in the management of patients and in the conduct and monitoring of randomised clinical trials. It is responsible for safeguarding the interests of trial participants, assessing the safety and efficacy of the interventions during the trial and for monitoring the overall conduct of the clinical trial. A detailed description of the NOCHOP DSMB and its duties can be found in the DSMB Charter, in the supplementary data file.

\section{Protection of participant privacy}

The NOCHOP study adheres to the privacy rules instituted by the Health Insurance Portability and Accountability Act of 1996 (HIPAA). All examination data are electronically transmitted using encryption software at the end of the visit, and all data are stored in firewallprotected servers. Data analysis will be performed in deidentified data sets (ie, sets that contain no participant identifiers, as defined by HIPAA). Moreover, only deidentified data sets will be shared using IRB-authorised procedures or (upon request) with the National Institutes of Health.

\section{Relevance and dissemination}

Our project seeks to examine the efficacy of a CHW intervention to improve the care of adult Hispanics with type 2 diabetes in Northern Manhattan. CHW interventions are rooted in over 30 years of culturally tailored public health service delivery in Hispanic communities. The acceptability of this approach to enhance healthcare delivery has been shown in Hispanic populations in numerous studies. An additional strength of the community-based design is that it will not further tax the already limited resources of health providers caring for low-income minority populations, such as inner-city clinics. In addition, this project will help characterise the specific components of a CHW intervention that lead to an improvement in clinically and socially relevant outcomes in this high-risk population. In doing so, NOCHOP is expected to make a substantial contribution to the ongoing national debate about the sustainability and optimal design of CHW programmes.

Contributors All authors edited the draft and contributed substantially to the manuscript; they all approved this submission. WP, JAT, SF, MM, MB and OC conceived of and designed the study. MM, JAT, JAL and SS participated in the design of the study. SF, MM and MB designed the community health workers intervention protocol, with input from WP, JAL and OC. MM and MB supervise the community health workers intervention, with assistance from WP and SF. JAT supervised the power analyses and wrote the data analyses section. SS and $\mathrm{JK}$ created the data entry system, under the supervision of JT; they will be responsible for performing the statistical analysis. JK programmed the Macros for power analyses. WP, OC and JAL designed and planned the evaluation visits. WP bears overall responsibility for the design, ethical conduct and publication of the study.

Funding This study was funded by the National Institute on Minority Health and Health Disparities and the National Institutes of Health, grant number P60 MD00020606.

\section{Competing interests None.}

Ethics approval Ethics approval was provided by the Columbia University Medical Center Institutional Review Board.

Provenance and peer review Not commissioned; internally peer reviewed.

\section{REFERENCES}

1. Mainous AG 3rd, Baker R, Koopman RJ, et al. Impact of the population at risk of diabetes on projections of diabetes burden in the United States: an epidemic on the way. Diabetologia 2007;50:934-40.

2. Harris MI, Eastman RC, Cowie CC, et al. Racial and ethnic differences in glycemic control of adults with type 2 diabetes. Diabetes Care 1999;22:403-8.

3. Harris MI. Racial and ethnic differences in health care access and health outcomes for adults with type 2 diabetes. Diabetes Care 2001;24:454-9.

4. Anon. Policy statements adopted by the Governing Council of the American Public Health Association, November 18, 1998. Am J Public Health 1999;89:428-50.

5. Mieres $\mathrm{JH}$, Phillips LM. The interdisciplinary approach to culturally tailored medical care: "Social networking" for decreasing risk: comment on "The effects of a nurse case manager and a community health worker team on diabetic control, emergency department visits and hospitalizations among urban African Americans with type 2 diabetes mellitus: a randomized controlled trial" and "Trial of family and friend support for weight loss in African American adults". Arch Intern Med 2009;169:1804-5.

6. Perez LM, Martinez J. Community health workers: social justice and policy advocates for community health and well-being. Am J Public Health 2008;98:11-14.

7. Babamoto KS, Sey KA, Camilleri AJ, et al. Improving diabetes care and health measures among hispanics using community health workers: results from a randomized controlled trial. Health Educ Behav 2009;36:113-26.

8. Brown SA, Garcia AA, Kouzekanani K, et al. Culturally competent diabetes self-management education for Mexican Americans: the Starr County border health initiative. Diabetes Care 2002;25:259-68.

9. Gary TL, Batts-Turner M, Bone LR, et al. A randomized controlled trial of the effects of nurse case manager and community health worker team interventions in urban African-Americans with type 2 diabetes. Control Clin Trials 2004;25:53-66.

10. Gary TL, Batts-Turner M, Yeh HC, et al. The effects of a nurse case manager and a community health worker team on diabetic control, emergency department visits, and hospitalizations among urban African Americans with type 2 diabetes mellitus: a randomized controlled trial. Arch Intern Med 2009;169:1788-94. 
11. Gary TL, Bone LR, Hill MN, et al. Randomized controlled trial of the effects of nurse case manager and community health worker interventions on risk factors for diabetes-related complications in urban African Americans. Prev Med 2003;37:23-32.

12. Lujan J, Ostwald SK, Ortiz M. Promotora diabetes intervention for Mexican Americans. Diabetes Educ 2007:33:660-70.

13. Lorig K, Ritter PL, Villa FJ, et al. Community-based peer-led diabetes self-management: a randomized trial. Diabetes Educ 2009;35:641-51.

14. Hawthorne K, Robles $\mathrm{Y}$, Cannings-John R, et al. Culturally appropriate health education for type 2 diabetes mellitus in ethnic minority groups. Cochrane Database Syst Rev 2008;(3):CD006424.

15. Sarkisian CA, Brown AF, Norris KC, et al. A systematic review of diabetes self-care interventions for older, African American, or Latino adults. Diabetes Educ 2003;29:467-79.

16. Lewin SA, Dick J, Pond P, et al. Lay health workers in primary and community health care. Cochrane Database Syst Rev 2005;(1): CD004015.

17. Swider SM. Outcome effectiveness of community health workers: an integrative literature review. Public Health Nurs 2002;19:11-20.

18. Bakken S, Lantigua RA, Busacca LV, et al. Barriers, enablers, and incentives for research participation: a report from the Ambulatory Care Research Network (ACRN). J Am Board Fam Med 2009;22:436-45.

19. Shea S, Weinstock RS, Teresi JA, et al. A randomized trial comparing telemedicine case management with usual care in older, ethnically diverse, medically underserved patients with diabetes mellitus: 5 year results of the IDEATel study. J Am Med Inform Assoc 2009; $16: 446-56$

20. White MJ, Stark JR, Luckmann R, et al. Implementing a computerassisted telephone interview (CATI) system to increase colorectal cancer screening: a process evaluation. Patient Educ Couns 2006:61:419-28.

21. Friedewald WT, Levy RI, Fredrickson DS. Estimation of the concentration of low-density lipoprotein cholesterol in plasma, without use of the preparative ultracentrifuge. Clin Chem 1972;18:499-502.

22. MacLaughlin EJ, Raehl CL, Treadway AK, et al. Assessing medication adherence in the elderly: which tools to use in clinical practice? Drugs Aging 2005;22:231-55.

23. Winston GJ, Barr RG, Carrasquillo O, et al. Sex and racial/ethnic differences in cardiovascular disease risk factor treatment and control among individuals with diabetes in the Multi-Ethnic Study of Atherosclerosis (MESA). Diabetes Care 2009;32:1467-9.

24. Blair SN, Haskell WL, Ho P, et al. Assessment of habitual physical activity by a seven-day recall in a community survey and controlled experiments. Am J Epidemiol 1985;122:794-804.

25. Thompson FE, Kipnis V, Subar AF, et al. Evaluation of 2 brief instruments and a food-frequency questionnaire to estimate daily number of servings of fruit and vegetables. Am J Clin Nutr 2000;71:1503-10.

26. Kroenke K, Spitzer RL, Williams JB. The PHQ-9: validity of a brief depression severity measure. J Gen Intern Med 2001;16:606-13.
27. Findley SE, Irigoyen M, See D, et al. Community-provider partnerships to reduce immunization disparities: field report from northern Manhattan. Am J Public Health 2003;93:1041-4.

28. Bar-Yam Y. Improving the effectiveness of health care and public health: a multiscale complex systems analysis. Am J Public Health 2006;96:459-66.

29. Whetten $\mathrm{K}$, Leserman $\mathrm{J}$, Whetten $\mathrm{R}$, et al. Exploring lack of trust in care providers and the government as a barrier to health service use. Am J Public Health 2006;96:716-21.

30. Konrad TR, Howard DL, Edwards LJ, et al. Physician-patient racial concordance, continuity of care, and patterns of care for hypertension. Am J Public Health 2005;95:2186-90.

31. Gunn PP, Fremont AM, Bottrell M, et al. The Health Insurance Portability and Accountability Act Privacy Rule: a practical guide for researchers. Med Care 2004:42:321-7.

32. Donner A. Approaches to sample size estimation in the design of clinical trials-a review. Stat Med 1984;3:199-214.

33. Sullivan LM, D'Agostino RB. Robustness and power of analysis of covariance applied to data distorted from normality by floor effects: homogeneous regression slopes. Stat Med 1996;15:477-96.

34. Hsieh FY. Sample size formulae for intervention studies with the cluster as unit of randomization. Stat Med 1988;7:1195-201.

35. Klar N, Donner A. The merits of matching in community intervention trials: a cautionary tale. Stat Med 1997;16:1753-64.

36. D'Agostino RB Sr. Controlling alpha in a clinical trial: the case for secondary endpoints. Stat Med 2000;19:763-6.

37. Koch GG. Discussion for 'Alpha calculus in clinical trials: considerations and commentary for the new millennium'. Stat Med 2000;19:781-4

38. Moye LA. Alpha calculus in clinical trials: considerations and commentary for the new millennium. Stat Med 2000;19:767-79.

39. Moye LA. Response to commentaries on 'Alpha calculus in clinical trials: considerations for the new millennium'. Stat Med 2000;19:795-9.

40. O'Neill RT. Commentary on 'Alpha calculus in clinical trials: considerations and commentary for the new millennium'. Stat Med 2000;19:785-99.

41. Proschan MA, Waclawiw MA. Practical guidelines for multiplicity adjustment in clinical trials. Control Clin Trials 2000;21:527-39.

42. Taylor A, West S, Aiken L. Loss of power in logistic, ordinal logistic and probit regression when an outcome variable is coarsely categorized. Educ Psychol Meas 2006;66:228-39.

43. Petkova E, Teresi J. Some statistical issues in the analyses of data from longitudinal studies of elderly chronic care populations. Psychosom Med 2002;64:531-47.

44. Liu X, Teresi JA, Waternaux C. Modelling the decline pattern in functional measures from a prevalent cohort study. Stat Med 2000;19:1593-606.

45. Gardiner JC, Luo Z, Roman LA. Fixed effects, random effects and GEE: what are the differences? Stat Med 2009;28:221-39.

46. Petkova E, Teresi J. Some statistical issues in the analyses of data from longitudinal studies of elderly chronic care populations. Psychosom Med 2002;64:531-47. 